\title{
Escape Kinetics of an Underdamped Colloidal Particle From a Cavity Through Narrow Pores
}

\author{
Shubhadip Nayak ${ }^{\dagger}$, Tanwi Debnath ${ }^{\ddagger}$, Shovan Das ${ }^{\dagger}$, Debajyoti Debnath ${ }^{\dagger}$, \\ And Pulak K. Ghosh ${ }^{\dagger *}$ \\ †Department of Chemistry, Presidency University, Kolkata 700073, India \\ ‡Department of Chemistry, University of Calcutta, Kolkata 700009, India \\ E-mail: pulak.chem@presiuniv.ac.in
}

\section{Supporting information}

\section{A1. Effective cavity width and space accessible to the particle's center of mass}

We simulate dynamics of particles' center of mass by considering point size particles in a cavity. Thus, in our model, cavity volume is the free space accessible to the center of mass. Numerical values of cavity width or radius and the bottle neck (pore size $\Delta$ ) reported in the manuscript are actually meant for the particle's center of mass. To clarify this issue, we schematically show the space available for the center of mass and the effective size of the cavity.

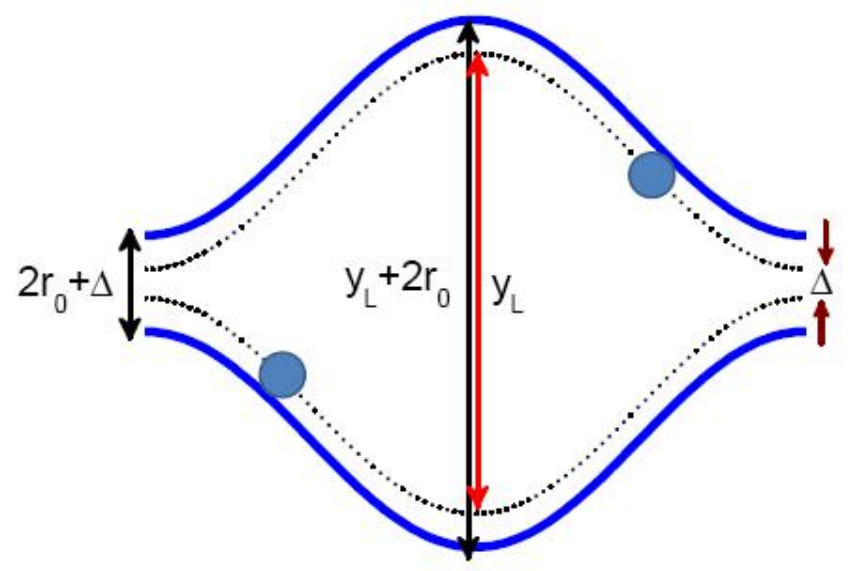

Figure S1: Schematics of a corrugated channel compartment depicting effective volume and free space accessible for the center of mass.

$\mathrm{Y} \mathrm{L}$ and $\Delta$ are the maximum cavity width and the pore size for the center of mass. Where as, $y_{L}+2 r_{0}$ and $2 r_{0}+\Delta$ are the corresponding width maximum and effective pore size for a finite size particles of radius $r_{0}$. 


\section{A2. Parameters for the Colloidal Brownian particle in the Langevin equations}

Consider Langevin equation for $\mathrm{x}$-component:

$$
m \ddot{x}=-\dot{x}+\sqrt{2 \gamma k_{B} T} \xi(t) \text { with }\langle\xi(t)\rangle=0 ; \quad\left\langle\xi(t) \xi\left(t^{\prime}\right)\right\rangle=\delta\left(t-t^{\prime}\right)
$$

Rearranging this equation:

$$
\ddot{x}=-\frac{\gamma}{m} \dot{x}+\sqrt{2\left(\frac{\gamma}{m}\right) \cdot\left(\frac{k_{B} T}{m}\right)} \xi(t)=-\frac{1}{\tau_{\gamma}} \dot{x}+\sqrt{2 \frac{\mathrm{v}_{t h}^{2}}{\tau_{\gamma}}} \xi(t) \ldots \ldots \text { (A1) }
$$

where $\mathrm{v}_{t h}=\sqrt{\frac{k_{B} T}{m}}$ and $\tau_{\gamma}=\frac{m}{\gamma}$

Thus free parameters for the colloidal particle in our simulation are the viscous relaxation time $\left(\tau_{\gamma}\right)$ and thermal velocity $\left(\mathrm{v}_{\mathrm{th}}\right)$. Alternatively one can choose thermal length $\left(l_{\text {th }}\right)$ and thermal velocity. It can be shown as follows:

$$
\ddot{x}=-\left(\frac{\mathrm{v}_{\text {th }}}{l_{\text {th }}}\right) \dot{x}+\sqrt{2\left(\frac{\mathrm{v}_{\text {th }}^{3}}{l_{\text {th }}}\right)} \xi(t)-\ldots-\ldots-\text { (A2) }
$$

where $\quad l_{\text {th }}=\tau_{\gamma} \mathrm{v}_{\text {th }}$

\section{A3. All the equations for mean exit time can be expressed in terms of thermal length $I_{\text {th }}$ and the thermal velocity $V_{\text {th }}$}

Examples:

Equation (2): $\tau_{e x}=\frac{2 \pi R}{\delta \theta} \sqrt{\frac{\pi m}{2 k_{B} T}}=\frac{2 \pi R}{\delta \theta} \sqrt{\frac{\pi}{2}} \frac{1}{\mathrm{v}_{\text {th }}}$

Equation (3):

$$
\begin{aligned}
& \tau_{e x}=\frac{\gamma R^{2}}{k_{B} T}\left(\ln \left[\frac{2(2 \pi-\delta \theta)}{\delta \theta}\right]+\frac{1}{4}\right)=\left(\frac{\gamma}{m}\right)\left(\frac{m}{k_{B} T}\right) R^{2}\left(\ln \left[\frac{2(2 \pi-\delta \theta)}{\delta \theta}\right]+\frac{1}{4}\right) \\
& =\frac{R^{2}}{\tau_{\gamma} \mathrm{v}_{t h}^{2}}\left(\ln \left[\frac{2(2 \pi-\delta \theta)}{\delta \theta}\right]+\frac{1}{4}\right)=\frac{R^{2}}{l_{t h} \mathrm{v}_{t h}}\left(\ln \left[\frac{2(2 \pi-\delta \theta)}{\delta \theta}\right]+\frac{1}{4}\right)
\end{aligned}
$$

Similarly, all the other equations for exit time can also be expressed in terms of thermal length $I_{\text {th }}$ and the thermal velocity $v_{\text {th. }}$. 


\section{A4: Tuning thermal velocity $v_{\text {th }}$ equivalent to re-scaling time}

Let us start with Langevin equation (A2)

$\kappa^{2} \ddot{x}=-\left(\frac{\kappa^{2} \mathrm{v}_{\text {th }}}{l_{\text {th }}}\right) \dot{x}+\sqrt{2\left(\frac{\kappa^{4} \mathrm{v}_{\text {th }}^{3}}{l_{\text {th }}}\right)} \xi(t) \quad$ [ both side are multiplied by $\kappa^{2}$ ]

Above equation can be rearranged as

$\frac{d^{2} x}{d\left(\frac{t}{\kappa}\right)^{2}}=-\frac{\left(\kappa \mathrm{v}_{\text {th }}\right)}{l_{t h}} \frac{d x}{d\left(\frac{t}{\kappa}\right)}+\sqrt{2\left(\frac{\left(\kappa \mathrm{v}_{\text {th }}\right)^{3}}{l_{t h}}\right)} \xi\left(\frac{t}{\kappa}\right) ;$ as $\xi(t)$ is $\delta$-corrected, $\xi\left(\frac{t}{\kappa}\right)=\sqrt{\kappa} \xi(t)$

This equation shows that increasing thermal velocity by a factor $\kappa$ is equivalent to re-scaling time as $t / \kappa$. This re-scaling can easily be checked in all equations for $\tau_{\mathrm{ex}}$ in the main text. Thus, increasing thermal velocity $\kappa$ times results in decrease of exit time by the factor $\kappa$. Further this re-scaling has been confirmed by the simulation results.
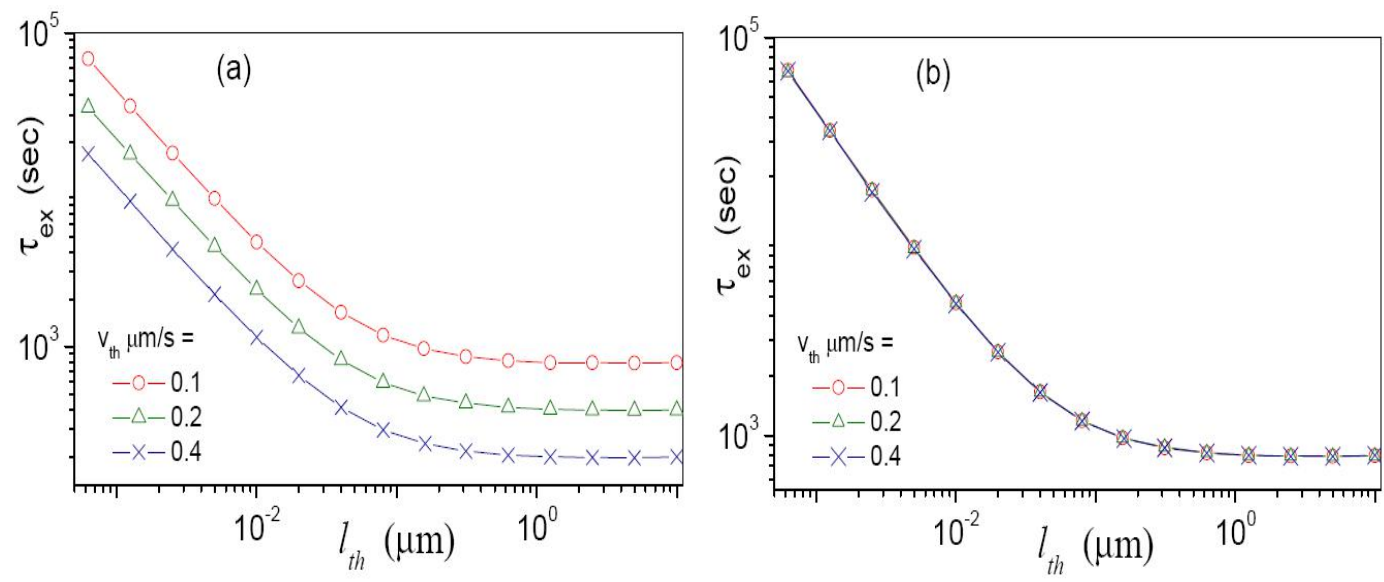

Figure S2: (a) Mean exit time from a circular cavity as a function of the thermal length for three different thermal velocities. (b) Shows that re-scaling time axis with appropriate factors leads to merging plots for different $v_{\text {th }}$ into a single one. Other simulation parameters: $R=1 \mu \mathrm{m} ; \Delta=0.1 \mu \mathrm{m}$

Figure S2(a) shows variation of exit time from a circular cavity with single opening as a function of thermal length $I_{\text {th }}$ for three different thermal velocity $v_{\text {th }}: 0.1 \mu \mathrm{m} / \mathrm{s}, 0.2$ $\mu \mathrm{m} / \mathrm{s}$, and $0.4 \mu \mathrm{m} / \mathrm{s}$. Now, re-scaling the mean exit time for the data set with $v_{\text {th }}=0.2$ $\mu \mathrm{m} / \mathrm{s}$ and $0.4 \mu \mathrm{m} / \mathrm{s}$ by the factor $1 / 2$ and $1 / 4$, respectively, results in merging all the three plots (shown in Figure S2(b)). These re-scaling is equivalent to changing velocity as $\mathrm{v}_{\mathrm{th}}=0.2$ to $\mathrm{v}_{\mathrm{th}}=0.1$ and $\mathrm{v}_{\mathrm{th}}=0.4$ to $\mathrm{v}_{\mathrm{th}}=0.1$, respectively. 


\section{A5: Accessibility of the inertial impact in the experiment}

In overdamped limits, it is known that the mean exit time is inversely proportional to the thermal length $I_{\text {th }}$ irrespective of the pore geometries. The deviation from this inverse relation is attributed to the inertial impact in the escape kinetics. Our simulation results [see Figure S3 ] show that inertial impacts become noticeable for the thermal lengths as small as $5 \mathrm{~nm}, 10 \mathrm{~nm}$ and $20 \mathrm{~nm}$ when pore widths are respectively $25 \mathrm{~nm}, 50 \mathrm{~nm}$ and $100 \mathrm{~nm}$ larger than the particle's diameter. Thus, impacts of underdamped motion in the escape kinetics can be accessed in experiments if the particle's thermal length is $5 \mathrm{~nm}$ or larger. The point up to which underdamping is meaningful (indicated by vertical arrows in the following Figure s3) depends on the exit window size and insensitive to the cavity size.

For a particle of size 1 micron and mass density $2 \mathrm{~g} / \mathrm{cm}^{3}, l_{\text {th }}$ is about $0.6 \mathrm{~nm}$ (water) and $30 \mathrm{~nm}$ (air). Again it can be tuned to some extent by changing particle size, mass density and temperature.

$$
l_{t h}=\frac{m}{6 \pi \eta_{\mathrm{v}} r_{0}} \sqrt{\frac{k_{B} T}{m}} \propto \frac{\sqrt{r_{0} \rho T}}{\eta_{\mathrm{v}}}
$$

Thus, the thermal length of micron size particles at room temperature can fall in the range $0.1 \mathrm{~nm}$ to $50 \mathrm{~nm}$ depending upon the medium viscosity and mass density of the colloids. This estimate shows that inertial effects can easily be accessed in the experiments.

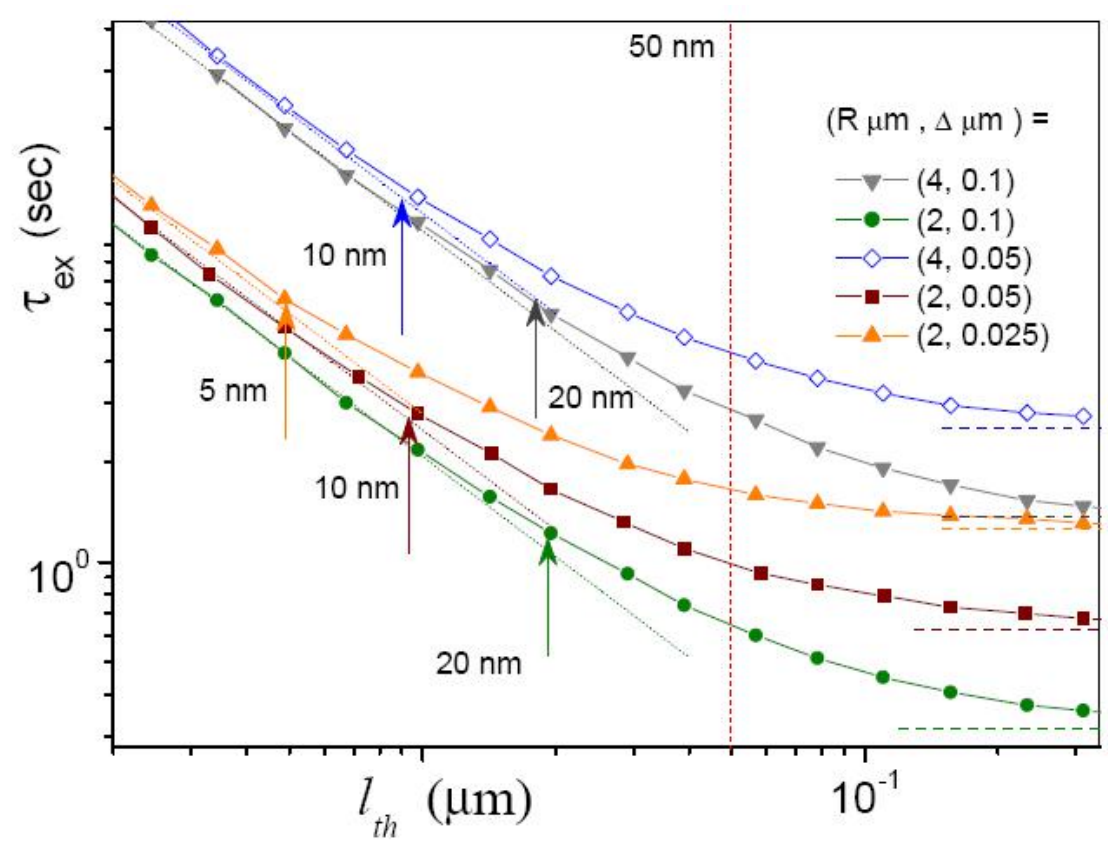


Figure S3: Thermal length versus exit time from a circular cavity. Note that this figure contains some data set common with Fig2. However for a closer look and to support our argument about experimental accessibility of inertial impacts, $l_{\text {th }}$ axis is cut to a shorter range. The vertical arrows depict the lower bounds of thermal length below which inertial effect can safely be ignored. The numerical values of the lower bounds given next to the arrows. Vertical dashed line at $l_{\mathrm{th}}=50 \mathrm{~nm}$ indicates appropriate upper bound of thermal length of micron size particles under physically feasible conditions. Our simulations use $v_{\text {th }}$ $=1000 \mu \mathrm{m} / \mathrm{s}$ and other parameters are mentioned in the legends. Dashed and dotted lines are analytical fitting based on the $\mathrm{Eq}(2)$ and (3), respectively.

Note that our observable is the escape time from a cavity which involves diffusion over a length much larger than the particle size. Our simulations assume cavities of radius 2 to 5 times of particle's size. More importantly, as mentioned earlier, the thermal length up to which inertial effect cannot be discarded is insensitive to the cavity size. Inside the cavity, the particle's ballistic motion persists up to the thermal length $I_{\text {th }}$ which is in practice much smaller than both the cavity and particle sizes. However, it can be comparable to the effective pore size (cavity bottleneck diameter minus particle diameter) in physically feasible conditions. Our simulation results in the above figure (also in the manuscript Figure 2) show that inertial impact persists in the escape kinetics until the particle's thermal length is as small as $20 \%$ of the effective pore size. This is due to the fact that the short-range ballistic motion, being relevant around exit windows, changes the course of the dynamics inside the cavity. Thus, conspicuously, short lived ballistic motions leave a noticeable impact on the escape kinetics. For experimental demonstration of inertial impacts thermal length need not be larger than the particle size.

Possible hydrodynamic impact: When a particle diffuses through a tube of uniform cross-section which is slightly larger than the particle's diameter, hydrodynamic effect can alter amplitude of the damping strength to some extent. The cases we studied are considerably different from this. For circular cavities or other types of cavities with sharp opening structures (see Figure S5), available free space between walls and the particle surface sharply increases as one moves a very little from the bottleneck center. For the case we mentioned above, the free space is about 25 to $100 \mathrm{~nm}$ only at the center of the bottleneck. Therefore, the impact of hydrodynamic effect here is not obvious at all. If there is something, it would be of very little amplitude. That effect cannot make inertial impact die out. Even if hydrodynamic impact makes damping 
strength double, particles thermal length will be reduced by a factor $1 / 2$. As per our simulation results, still inertia effects will remain accessible under experimental conditions.

\section{A6: Effects of initial conditions on the exit time}

Here we compare the mean exit time for two different initial conditions:

(i) Particles are placed at the center of the cavity with Maxwellian distribution of velocity at $\mathrm{t}=0$

(ii) Initial positions are randomly chosen within the cavity with Maxwellian distribution of velocity at $\mathrm{t}=0$

Figures S4 show simulation results of exit times from various types of cavity versus thermal length for different initial conditions.

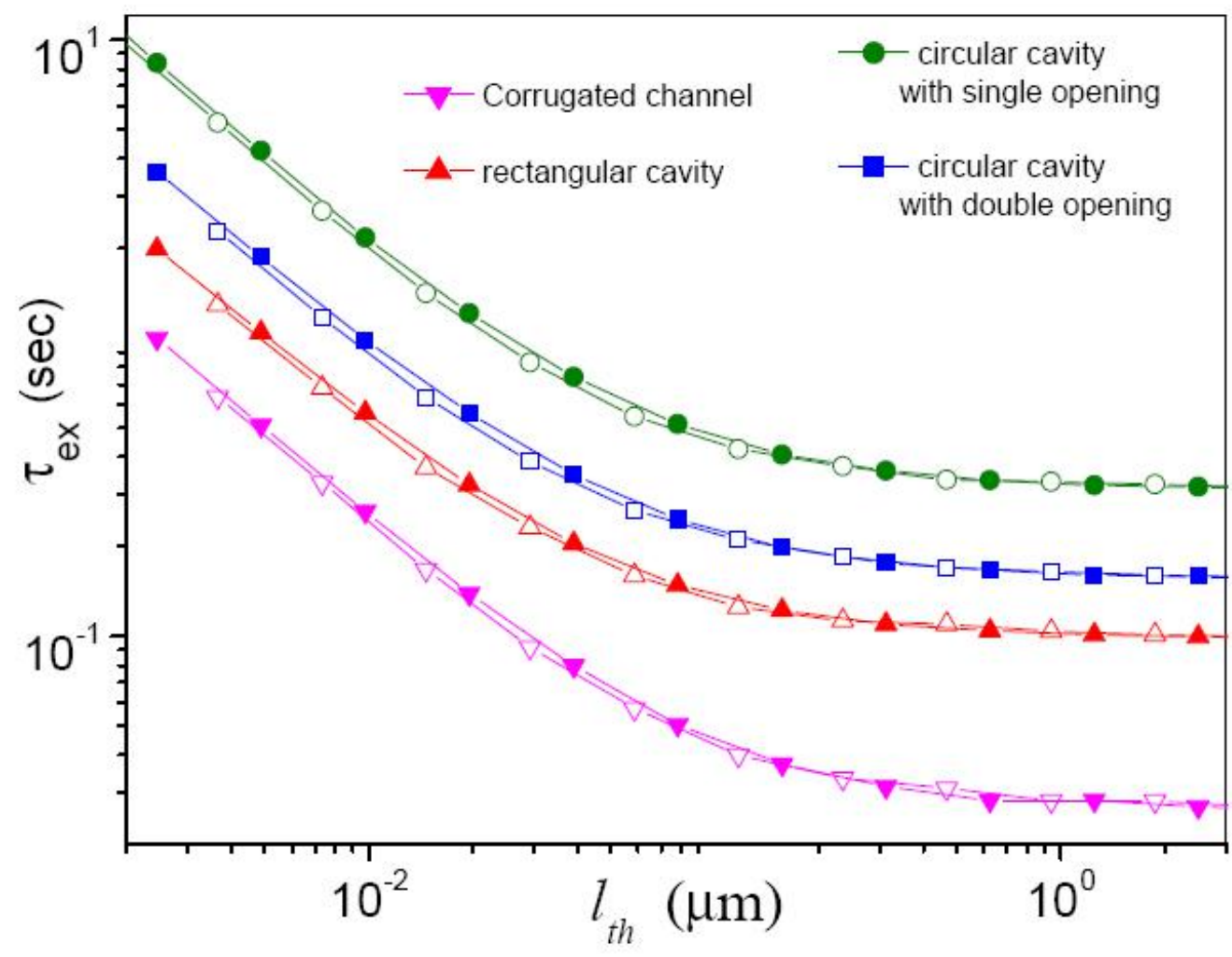

Figure S4: Mean exit times from different types of cavities (as mentioned in the legends) as a function of the thermal length for two types of initial conditions: (i) particles are initially placed at the center of the cavity (solid symbols) and (ii) initial position are randomly chosen within the cavity. For both cases, we assume Maxwellian distribution of initial velocity. Simulation parameters: $\mathrm{v}_{\mathrm{th}}=1000 \mu \mathrm{m} / \mathrm{s}, \mathrm{x}_{\mathrm{L}}=$ $\mathrm{y}_{\mathrm{L}}=2 \mu \mathrm{m}$ (for rectangular cavity and compartment of corrugated channel with $\eta=2$ ) $\mathrm{R}=2 \mu \mathrm{m}$ (for circular cavity with single or double openings), and $\Delta=0.1 \mu \mathrm{m}$. 
The above figure clearly shows that exit times are almost equal for the both types of initial conditions. To understand these results we consider the case of a circular cavity with a single opening. Similar line of reasoning can be used for other types of cavities. We analyse the underdamped and overdamped limits, separately.

\section{Underdamped limit:}

We recourse Eq. (2) to obtain the following expression for the mean exit time when the particle is initially placed at a distance $d$ from the boundary wall,

$$
\tau_{e x}=\left[\frac{2 \pi R}{\delta \theta}+\langle(d-R)\rangle\right] \sqrt{\frac{\pi m}{2 k_{B} T}} .
$$

Where, $<. .>$ indicates averaging over initial positions. For a small pore size $\delta \theta$,

$$
\frac{2 \pi R}{\delta \theta}>>|\langle(d-R)\rangle|
$$

Thus, the exit time becomes almost equal for both types of initial conditions.

\section{$\underline{\text { Overdanmped limit }}$}

In the overdamped situation, the exit time is insensitive to the initial position unless the particle is placed at the boundary layer near the exit window [see, Holcman, D.; Schuss, Z. Stochastic Narrow Escape in Molecular and Cellular Biology, Springer: New York, 2015; page 105]. Thus, the exit time for both types of initial condition is almost equal when the exit window is small. Following are the analytic expressions of mean exit time for both types of initial conditions [1]:

(i) When particles are placed at the center of the cavity at $t=0$

$$
\tau_{e x}=\frac{\gamma R^{2}}{k_{B} T}\left(\ln \left[\frac{2(2 \pi-\delta \theta)}{\delta \theta}\right]+\frac{1}{4}\right) .
$$

(ii) When particles initial position are randomly chosen within the cavity

$$
\tau_{e x}=\frac{\gamma R^{2}}{k_{B} T}\left(\ln \left[\frac{2(2 \pi-\delta \theta)}{\delta \theta}\right]+\frac{1}{8}\right) .
$$

It is apparent from the above expression that the mean exit time is almost equal for the both cases when the pore size is very small. 


\section{A7: Schematic for periodic channels}

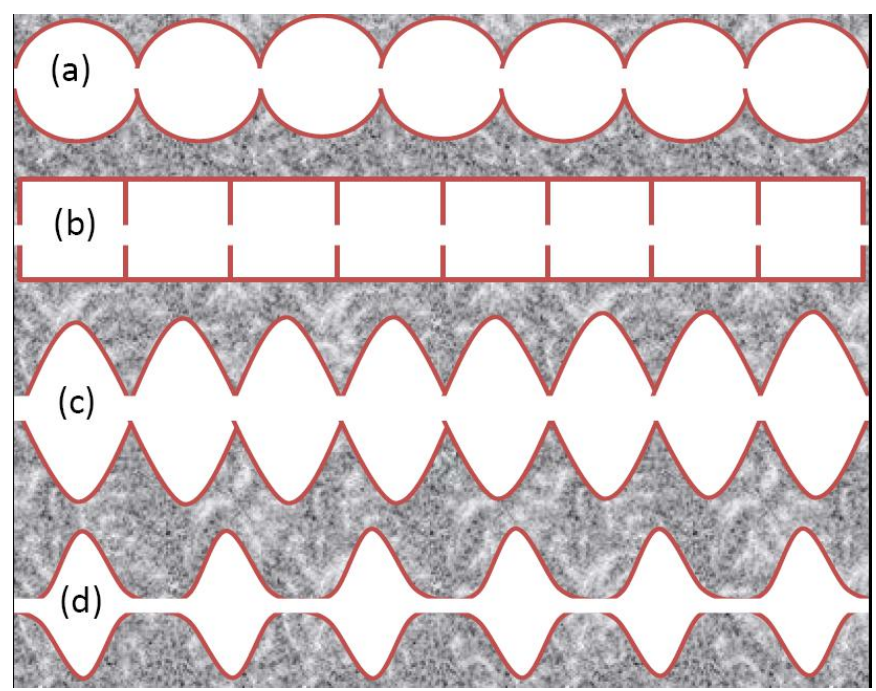

Figure S5: Schematics of periodic channel formed by connecting various types of cavities [(a) circular, (b) rectangular, (c) and (d) corrugated channel compartments with different pore structures ] present in Fig. 1 of the main text.

\section{References:}

[1] Holcman, D.; Schuss, Z. Stochastic Narrow Escape in Molecular and Cellular Biology, Springer: New York, 2015; page 105 